\title{
Video Article \\ Continuous Theta Burst Stimulation of the Posterior Medial Frontal Cortex to Experimentally Reduce Ideological Threat Responses
}

\author{
Colin Holbrook ${ }^{1}$, Chelsea L. Gordon ${ }^{1}$, Marco lacoboni ${ }^{2}$ \\ ${ }^{1}$ Department of Cognitive and Information Sciences, University of California, Merced \\ ${ }^{2}$ Department of Psychiatry and Biobehavioral Sciences, University of California, Los Angeles
}

Correspondence to: Colin Holbrook at cholbrook01@ucla.edu

URL: https://www.jove.com/video/58204

DOI: doi:10.3791/58204

Keywords: Behavior, Issue 139, Ethnocentrism, religiosity, transcranial magnetic stimulation, continuous theta burst stimulation, posterior medial frontal cortex, threat

Date Published: 9/28/2018

Citation: Holbrook, C., Gordon, C.L., lacoboni, M. Continuous Theta Burst Stimulation of the Posterior Medial Frontal Cortex to Experimentally Reduce Ideological Threat Responses. J. Vis. Exp. (139), e58204, doi:10.3791/58204 (2018).

\section{Abstract}

Decades of behavioral science research have documented functional shifts in attitudes and ideological adherence in response to various challenges, but little work to date has illuminated the neural mechanisms underlying these dynamics. This paper describes how continuous theta burst transcranial magnetic stimulation may be employed to experimentally assess the causal contribution of cortical regions to threat-related ideological shifts. In the example protocol provided here, participants are exposed to a threat prime-an explicit reminder of their own inevitable death and bodily decomposition-following a downregulation of the posterior medial frontal cortex (pMFC) or a sham stimulation. Next, disguised within a series of distracter tasks, participants' relative degree of ideological adherence is assessed-in the present example, with regard to coalitional prejudice and religious belief. Participants for whom the pMFC has been downregulated exhibit less coalitionally biased responses to an immigrant critical of the participants' national in-group, and less conviction in positive afterlife beliefs (i.e., God, angels, and heaven), despite having recently been reminded of death. These results complement prior findings that continuous theta burst stimulation of the pMFC influences social conformity and sharing and illustrate the feasibility of investigating the neural basis of high-level social cognitive shifts using transcranial magnetic stimulation.

\section{Video Link}

The video component of this article can be found at https://www.jove.com/video/58204/

\section{Introduction}

This paper presents a recently developed method for experimentally neuromodulating ideological threat-reactions, with a particular focus on nationalistic bias and religiosity ${ }^{1}$. Importantly, however, the procedure presented in what follows should be taken as an illustrative token of a promising general approach to the study of the neural substrate of high-level social and ideological cognition (e.g., with regard to normative judgments, political attitudes) using transcranial magnetic stimulation (TMS). To theoretically situate this 'proof-of-concept' example, prior work on links between threat-detection and ideological investment, including plausible neural correlates of these effects, is briefly reviewed.

\section{Threat and Ethnocentrism}

People live in, and sometimes die for, social groups ${ }^{2}$. By assorting into coalitions, individuals benefit from shared access to both knowledge and material resources. Because sharing valued material or informational resources renders people vulnerable, individuals are incentivized to calculate whether others are likely to reciprocate or abuse their generosity ${ }^{3}$. Categorizing another person as sharing an investment in an in-group is thought to privilege coordination between fellow in-group members by enhancing mutual care and trust. This group-favoring ethnocentrism can also lead to a negative valuation of those perceived to be aligned with out-groups-and hence to be unreliable if not overtly antagonistic and, therefore, undeserving of in-group resources ${ }^{4,5}$. Under contexts of conflict, group prejudice appears not only to discourage cooperation but to motivate aggression against individuals perceived to belong to or sympathize with enemy coalitions ${ }^{6}$. If, over deep time, in-group favoritism advances reproductive fitness ${ }^{7,8}$, then the mind may have been shaped by selection to support ethnocentrism ${ }^{9,10}$, particularly under contexts of threat ${ }^{11,12}$. Consistent with this functional interpretation of ethnocentrism, the extent to which individuals express commitment to group ideologies has been observed to increase following primes of threat ${ }^{13,14,15}$. Although social scientists have studied the influence of threats on ideological commitment for decades, only recently has attention turned to the brain mechanisms at work ${ }^{16,17,18,19,20}$. In the present protocol, a brain region previously associated with low-level problem solving (e.g., updating motor responses to obtain a reward) is demonstrated to contribute to facultative shifts in ideological beliefs.

\section{Threat, Ideological Commitment, and the Posterior Medial Frontal Cortex}

The pMFC includes the dorsal anterior cingulate cortex (dACC) and the dorsomedial prefrontal area anterior to the supplementary motor cortex (dmPFC). The pMFC has been implicated in an array of reactions to negative stimuli ${ }^{21,22,23}$. The pMFC contributes to the detection of 
discrepancies between current and preferred conditions, as well as to subsequent decision-making adjustment of behavior to reduce such discrepancies ${ }^{24,25,26}$. For example, the $\mathrm{dACC}$ has been implicated in relatively low-level cognitive control functions such as those measured in the Stroop, Flanker, divided attention, or Go/No-Go tasks ${ }^{25}$. Similarly, at a higher level of abstraction, the dACC component of the pMFC is hypothesized to evoke intensified expressions of ideological commitment to moral or cultural values following exposure to threats (e.g., reminders of uncertainty or death) ${ }^{17,18}$. Cues of the concept of death have been found to similarly trigger activity in the dmPFC $\mathrm{C}^{27,28}$ and to intensify ideological expressions (e.g., national identification, punishment of norm-violators) ${ }^{14}$. Cues of social isolation have likewise been observed to heighten ethnocentrism ${ }^{12}$ and to activate the $\mathrm{dACC}^{29}$.

The dmPFC component of the pMFC is a particularly plausible inhibitor of prosociality toward out-group members, as the dmPFC is considered important to modulating the treatment of self versus other during social decision-making ${ }^{30,31}$. A growing literature suggests that human prosocial inclinations-most pronounced toward fellow in-group members, all else being equal-may partially derive from a tendency for feelings and behaviors to activate similar neural systems, whether originating in the self or in another ${ }^{32}$. The extent of this self-other neural resonance has been found to predict prosocial behavior ${ }^{33,34,35}$. Consistent with the role of coalitional bias in moderating prosociality, neural resonance and related prosocial behaviors are influenced by factors such as group identity ${ }^{36,32}$. The modulation of prosociality contingent on group identification may owe to mechanisms of inhibitory prefrontal cognitive control involving the dmPFC, as the dmPFC is important for the tonic control of spontaneous imitation ${ }^{37,30}$, as well as for shifting between the perspectives of self and other ${ }^{38}$. Most compellingly, downregulating the dmPFC causes greater financial sharing behavior ${ }^{39}$, directly implicating the dmPFC as inhibiting prosociality, plausibly including the suppression of prosociality on the basis of out-group affiliation. These relatively high-level social functions of the dmPFC may be understood as manifestations of a larger role of the dmPFC in various cognitive control functions ${ }^{40}$. For example, experimentally upregulating the dmPFC was recently shown to enhance impulse control in an asocial delay-discounting paradigm in which participants who postpone immediate reward receive greater future reward $^{41}$.

The pMFC complex appears responsive to cues of the presence of various sorts of social discrepancies, and pMFC activity predicts behavioral shifts designed to reduce said discrepancies ${ }^{42}$. For instance, pMFC activity correlates with preference changes in a manner that appears to reduce cognitive dissonance ${ }^{43,44,45}$ or to heighten social conformity following evidence that a person's opinions deviate from the group consensus ${ }^{46,47}$. In a demonstration of the causal role of the pMFC in enabling such dynamics, the experimental downregulation of pMFC activity via TMS has been shown to decrease social conformity despite cues of disagreement with the group ${ }^{48}$. In sum, upon detection of relatively low-level problems such as receiving a negative outcome in a motor task ${ }^{24}$, or relatively high-level problems such as a deviation between a person's stated views and those of his/her peers, the pMFC appears involved in the activation of networks that coordinate problem-relevant responses ${ }^{23,47,49}$

Considered together, the overall pattern of results implicates the pMFC as part of neurobiological architecture evolved to manage challenges spanning various low-level and high-level domains. Accordingly, when participants are presented with an out-group member's critique of their group, the pMFC would hypothetically be involved in detecting this conflict and with coordinating a typical response: derogation of that out-group critic and their ideas. By the same logic, the pMFC is predicted to help enable individuals confronted with their own mortality to amplify their belief in a pleasant afterlife. If so, then participants for whom the pMFC complex has been downregulated may be expected to evince less derogation of critical out-group members and less religious belief following reminders of the inevitability of death.

\section{Downregulating Targeted Cortical Areas Via Continuous Theta Burst Stimulation}

Theta burst stimulation (TBS) is a form of patterned TMS. TMS stimulates the brain non-invasively by producing a rapidly varying magnetic field over the stimulated subject's scalp. This rapidly varying magnetic field induces electric currents in the brain, which in turn lead brain cells
to fire ${ }^{50,51,52}$. In this manner, the manipulation of targeted brain regions via TMS allows research to move past the correlational discoveries of traditional brain mapping methods employing neuroimaging. By stimulating a given brain region and, thereby, decreasing or increasing its activity, causal inferences about the relevance of that region on a variety of behavioral tasks may be inferred.

TBS protocols have been modeled from repetitive electrical stimulation protocols that induced long-term potentiation (LTP) or long-term depression (LTD) in animal studies ${ }^{53}$. Continuous theta burst stimulation (cTBS), which consists of $50 \mathrm{~Hz}$ triplets of pulses delivered at $5 \mathrm{~Hz}$ for $40 \mathrm{~s}$, for a total of 600 pulses, has an effect similar to LTD, reducing the activity in the stimulated area for an estimated period of at least 1 hour. Intermittent TBS (iTBS) consists of the same pattern of bursts at the same frequency of cTBS. However, in iTBS, the subject is stimulated for $2 \mathrm{~s}$ at a time, which is repeated every $10 \mathrm{~s}$ for $190 \mathrm{~s}$ (totaling 600 pulses, as in cTBS). iTBS has an effect similar to LTP, enhancing the activity in the stimulated area for a period of time comparable to cTBS. Whereas the cTBS method highlighted here can reduce ideological threat-responses, theoretically, iTBS may heighten ideological threat-responses.

The protocol that follows details methods recently used to experimentally downregulate group bias and religious belief with cTBS ${ }^{1}$, in the hope that researchers interested in alternate modes of ideological threat-response might replicate these effects and/or modify this general approach for their own purposes (e.g., substituting alternate threat primes and/or judgment outcomes, or by adding a control stimulation site).

\section{Protocol}

All methods described in what follows have been approved by the Office of the Human Research Protection Program (OHRPP) of the University of California, Los Angeles.

\section{Pre-experiment Steps}

1. During recruitment, prescreen participants to ensure that they have no medical concerns, no history of neurological or psychological disorders, and no other disqualifying conditions for undergoing TMS, such as using a pacemaker, having any metal implants other than dental fillings, suffering a serious medical illness, taking anti-depressant or anti-psychotic medications, having a personal or family history of seizure disorder, or pregnancy. 
2. When prescreening (e.g., via phone), also ask questions regarding the prospective participant's political orientation, US citizenship, religiosity, and ethnicity.

1. Exclude non-US citizens or individuals who identify as 'extremely liberal' from the study to ensure that the participants will have a negative response to criticisms of the US (e.g., from a Latino immigrant, as has similarly been done in prior studies of intergroup bias) ${ }^{12,54,55}$.

2. Exclude those who identify as either 'extremely religious' or as 'atheist/agnostic' to ensure that participants will introspectively consider their degree of religious belief rather than answer reflexively on the basis of hardened convictions or habitual responses.

3. Exclude individuals who self-identify as 'Hispanic/Latino' in advance of the study toensure that the participants view the immigrant characters in the group bias measure as out-group members.

3. Continue recruitment until there are at least 20 participants who satisfy the screening criteria in each cell of the design.

4. Obtain written consent after thoroughly explaining the experiment, including what TMS is and how it works, upon arrival of the prospective participants at the laboratory.

\section{2. cTBS Procedure}

1. Seat the participant in a comfortable position and fit a grid-marked swim cap to his/her head. Use earplugs if needed to increase the comfort of the subject. Attach two differential parallel-bar EMG-recording electrodes, centered over the belly of the tibialis anterior muscle, after cleaning the skin over the muscle thoroughly. Attach a third, ground electrode to the skin over a bone somewhere else on the hand or arm.

2. On the swim cap, measure and mark the center location $(\mathrm{Cz})$ on the subject's head.

1. If using neuronavigation (recommended), launch the neuronavigation software and follow the software's procedure for localizing the subject's head. Have the subject rest comfortably and begin recording the EMG electrode output to software that will filter and display the signal.

3. Perform thresholding on the primary motor cortex. With a double-cone coil $(110 \mathrm{~mm})$, place the center of the coil over the motor cortex, held tangential to the scalp surface. Apply single-pulse TMS at $\mathbf{5 0 \%}$ of the maximum stimulus output (MSO) and observe whether a motor-evoked potential (MEP) was present in the EMG signal following the stimulation.

1. If no MEP is seen following the stimulation, reposition the coil $1 \mathrm{~cm}$ away in any direction and try the stimulation again. Wait at least $6-$ $10 \mathrm{~s}$ between stimulations in order for the neurons to completely recover. Continue moving the coil $1 \mathrm{~cm}$ at a time, marking on the cap the stimulation sites that result in an MEP of $50 \mathrm{mV}$ or greater.

2. If no MEPs are seen after trying many locations, increase the stimulation intensity by $5 \%$ at a time, until MEPs are observed.

3. Multiple nearby locations on the grid may elicit reliable MEPs. If this is the case, stimulate each of these locations at intensities decreasing by $1 \%$ at a time, until only one location remains that elicits reliable MEPs.

4. To determine the active motor threshold (aMT), have the subject slightly contract the target muscle. Stimulate the located region for 10 repetitions, separated by $\sim 7 \mathrm{~s}$, at decreasing intensities, until a corresponding observable twitch in the hand muscle no longer occurs for $50 \%$ of the stimulations (5 out of 10 ). The threshold is the lowest intensity that elicits $5 / 10$ twitches.

1. Navigate the coil to the specified location on the cap corresponding to the brain region of interest. If the double-cone coil to be used has a directionality of current flow, and if the target region is lateralized, orient the coil laterally so that the current flow is directed toward the hemisphere to be stimulated (e.g., a rightward current flow for a right hemisphere target) ${ }^{56,57}$.

1. If using neuronavigation, locate the coordinates for the region of interest (pMFC) at Montreal Neurological Institute (MNI) coordinates $[8,16,52]$ and mark this point on the system. Use the guiding software to target the region with the coil.

2. If neuronavigation is unavailable due to logistical or financial constraints (as was the case in the example experiment), determine the location of the pMFC for each subject's head using the international $10-20$ system $^{58}$. Place the coil $3.75 \mathrm{~cm}$ anterior to the motor cortex.

5. Apply cTBS as follows: three pulses at $50 \mathrm{~Hz}$ repeated at $200 \mathrm{~ms}$ intervals for $40 \mathrm{~s}$, totaling at 600 pulses.

6. If the participant is in the sham group, apply cTBS at only $10 \%$ of the maximal stimulation output.

\section{Survey Tasks}

1. Seat participants alone, in a private setting, at a desktop computer to perform the computer-mediated survey tasks.

2. Remind participants that their responses will be anonymous, confidential, and inaccessible to the research assistant, particularly to the extent that the target judgments (e.g., group bias and religious belief) are likely to raise self-presentation concerns (e.g., to appear patriotic, or not to appear prejudiced) which might obscure the effect of the cTBS manipulation.

3. Present participants with filler tasks for $\mathbf{1 0}$ min before starting the main task, as the maximal effect of cTBS begins 5-10 min after the stimulation ${ }^{53}$, and in order to minimize demand effects.

1. Administer the Multiple Source Interference Task ${ }^{59}$ (MSIT) (or a comparable distracter), as this task is highly cognitively demanding and requires approximately $10 \mathrm{~min}$.

2. Next, present a set of ostensibly unrelated survey tasks, beginning with two further filler distracters: challenge the participants to estimate the number of colors present in images of jellybeans and seashells that have been converted to greyscale.

4. Instantiate the threat context. If the ideological shifts of theoretical interest pertain to responses to death (as in this example study), ask participants to write two brief passages on the subject of their mortality ${ }^{60}$ by saying: (a) "Please briefly describe the emotions that the thought of your own death arouses in you" and (b) "Please jot down, as specifically as you can, what you think will happen to your body as you physically die and once you are physically dead." 
5. Following the threat-induction task, administer the Positive and Negative Affect Schedule - Expanded Form (PANAS-X) ${ }^{61}$ to allow selfreporting of potential effects of the cTBS intervention on emotional responses to the threat induction, as well as to distract the participants from the death prime encountered earlier in the protocol ${ }^{55}$.

6. Next, administer the relevant ideological judgment tasks.

NOTE: In the example given here, group bias and religious conviction were assessed. If more than two measures are employed, present them in a counterbalanced order.

1. To assess group bias with regard to ideological conflict, present participants with two essays ostensibly written by immigrants to the United States from Latin America (counterbalanced order) and ask participants to evaluate the authors and their arguments ${ }^{62}$. Note that there are intentional grammatical errors included in both essays which should not be corrected.

1. Present the "Pro-U.S." essay:

The first thing that hit me when I came to this country, was the incredible freedom people had. Freedom to go to school, freedom to work any job you want. In this country people can go to school and train for the job they want. Here anyone who works hard can make their own success. In my country most people live in poverty with no chance of escape. In this country people have more opportunity for success than any other and success does not depend on the group belong to. While there are problems in any country, America truly is a great nation and I don't regret my decision to come here at all."

2. Present the"Anti-U.S." essay:

When I first came to this country I believed it was the "land of opportunity" but I soon realized this was only true for the rich. The system here is set up for the rich against the poor. All people care about here is money and trying to have more than other people. This no sympathy for people. It's all one group putting down others and nobody cares about the foreigners. The people only let foreigners have jobs like pick fruit or wash dishes because no American would do it. Americans are spoiled and lazy and want everything handed to them. America is a cold country that is unsensitive to needs and problems of foreigners. It thinks it's a great country but its not."

3. After presenting each essay, ask participants to rate their agreement with six statements using an 8-point Likert scale (1= 'Strongly Disagree'; 8 = 'Strongly Agree'): (i) "I like the person who wrote this", (ii) "I think this person is intelligent", (iii) "This is the kind of person I would like to work with", (iv) "I think this person is honest", (v) "I agree with this person's views", and (vi) "I think this person's opinions of America are true."

4. Assess these responses for reliability and, if sufficiently reliable, average them. To explore potentially distinct effects of cTBS on personal affiliation (items I - iv) versus ideological agreement (items $v$ and vi), average them to create submeasures (see Figure 1).

2. Measure religious conviction according to a modified version of the Supernatural Belief Scale ${ }^{63}$ (SBS) in which two distinct subscales tap positive and negative aspects of Western religious belief, mirroring the positive and negative valence of the two essays in the group bias measure.

1. Present the SBS items in random order (positive scale: $\mathrm{i}$ - iii; negative scale: iv - vi), rated according to the same scale employed in the group bias measure: (i) "There exists an all-powerful, all-knowing, loving God"; (ii) "There exist good personal spiritual beings, whom we might call angels"; (iii) "Some people will go to heaven when they die"; (iv) "There exists an evil personal spiritual being, whom we might call the Devil"; (v) "There exist evil, personal spiritual beings, whom we might call demons", and (vi) "Some people will go to hell when they die."

2. Assess the responses for each subscale for reliability and average them if sufficiently reliable.

\section{Representative Results}

In the example study, the final sample consisted of 38 participants $\left(58 \%\right.$ female, $M_{\text {age }}=20.9$ years, $\left.S D=2.67\right)$. About $36.8 \%$ of the participants identified as White, $36.8 \%$ as East Asian, $13.2 \%$ as South Asian, $7.9 \%$ as Middle Eastern, and $5.3 \%$ as Other. As intended, the sample was politically moderate $(M=4.68, \mathrm{SD}=1.51 ; 1=$ "Extremely Liberal"; 5 = "Moderate"; 9 = "Extremely Conservative").

Preliminary ANOVA tests detected a significant effect of the order of essay presentation on ratings of the "anti-US" immigrant, $F(1,36)=5.30$, $p=0.027, \eta_{p}^{2}=0.13,95 \%$ confidence interval $(\mathrm{Cl})[-2.07,-.13]$, with no order effect observed for ratings of the "pro-US" immigrant, $p=0.74$. Accordingly, the essay order was included as a covariate in subsequent analyses. (Follow-up tests confirmed that controlling for order does not alter the overall pattern of results.) Such order effects may occur using this measure of group bias and should be routinely checked for as potential sources of noise.

As predicted, cTBS of the pMFC increased positive evaluations of the critical immigrant author, who was rated $28.5 \%$ more positively in the TMS condition $(M=4.10, S D=1.66)$ than in the control condition $(M=2.93, S D=1.22), F(1,35)=7.01, p=0.012, \eta_{p}^{2}=0.17,95 \% \mathrm{Cl}[-2.06$ -0.27]. Also consistent with the hypothesis that the pMFC reacts to ideological conflict, ratings of the pro-US author (who posed no ideological threat) were an average of $8.2 \%$ higher in the cTBS condition $(M=5.90, S D=.87)$ than in the sham condition $(M=5.42, S D=1.17)$, a difference that was not statistically significant, $F(1,36)=2.09, p=0.157, \eta_{p}^{2}=0.06,95 \% \mathrm{Cl}[-1.16,0.20]$. Remarkably, exploratory tests revealed that the effects of cTBS were equivalent with respect to both the ratings of the immigrants' personal qualities and the endorsement of their harshly critical arguments, attesting to an effect of the CTBS manipulation on the intensity of the participants' ideological investment in nationalistic values, rather than an effect limited to interpersonal amity (see Figure 1). This finding suggests that applications of this cTBS method addressing forms of ideological adherence relatively unrelated to social affiliation may be attenuated. 
Also consistent with predictions, participants who received cTBS reported an average of $32.8 \%$ less positive religious conviction $(M=3.05$, $S D=$ 1.92) relative to the sham participants $(M=4.54, S D=2.26), F(1,36)=4.80, p=0.035, \eta_{p}^{2}=0.12,95 \% \mathrm{Cl}[0.11,2.87]$. Participants in the cTBS condition also reported a less negative religious conviction $(M=2.84, S D=1.89)$ relative to the sham participants $(M=3.98, S D=2.50)$, but this difference did not reach statistical significance, $F(1,36)=2.52, p=0.122, \eta_{p}^{2}=0.07,95 \% \mathrm{Cl}[0.32,2.60]$. This result underscores the apparent relevance of the pMFC to recruiting ideological stances (e.g., positive religious beliefs) pertinent to particular problems (e.g., mortality), rather than to ideological adherence in general, as heaven presents a better solution than hell to the problem of death.

There were no apparent effects of condition on any of the 11 PANAS-X affect subscales ( $p s$ 0.09-0.92). Participants in both conditions reported moderately positive affect (cTBS: $M=2.44, S D=0.61$; Sham: $M=2.37, S D=0.75$ ) and low levels of negative affect $(\mathrm{cTBS}: M=1.22, S D=$ 0.27 ; Sham: $M=1.43, S D=0.51$ ). The null effects of cTBS on self-reported emotion suggest that the findings with regard to group bias or religiosity were not driven by changes in emotional reactivity to the reminder of death.

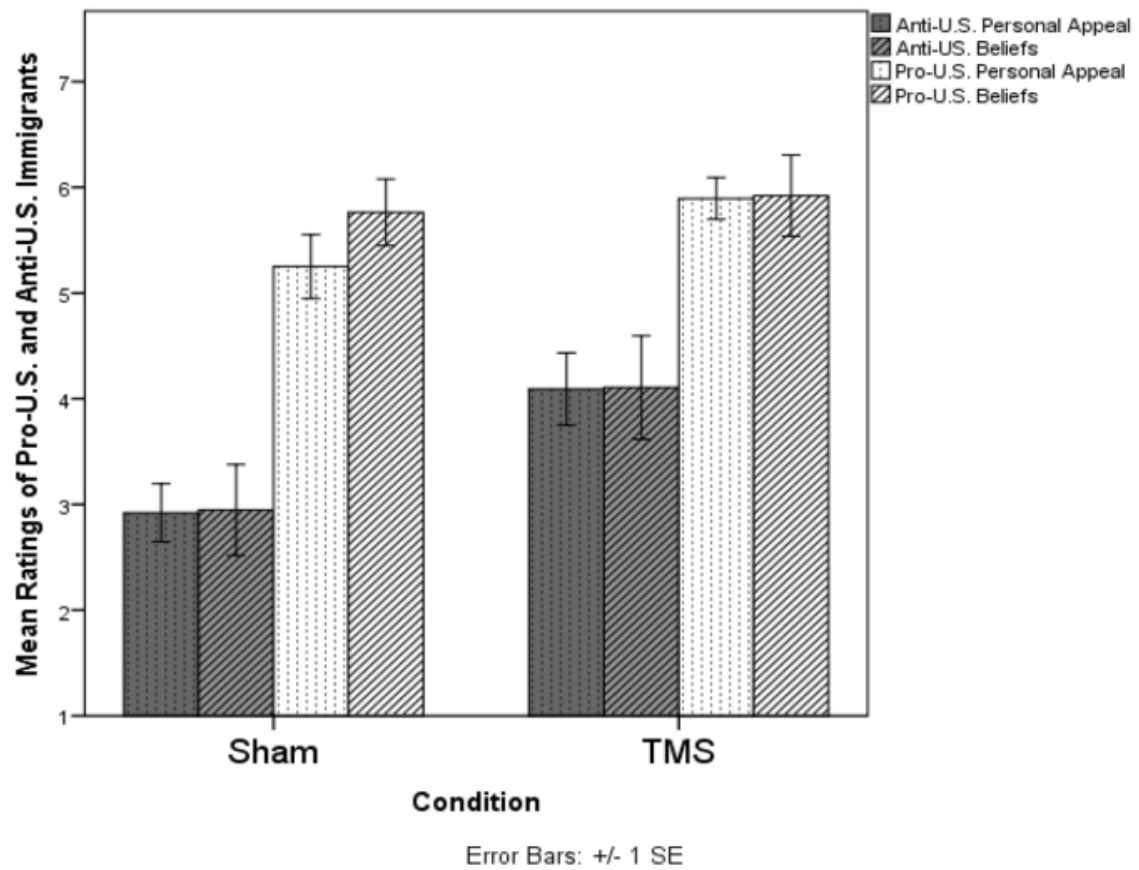

Figure 1: Effects of cTBS on US citizens' endorsement of the personal appeal and arguments of pro-US and anti-US immigrants. Please click here to view a larger version of this figure.

\section{Discussion}

Downregulating the pMFC via cTBS significantly reduced bias toward an out-group member critical of the US and avowed religious belief in the aftermath of a vivid reminder of death, consistent with both the hypothesized role of the pMFC in ideological threat-reactions and with the promise of TMS as a method for experimentally manipulating ideological cognition. While encouraging and consonant with a substantive background literature, the results of any single study should be considered to be highly preliminary. In addition, the cTBS methods employed here should be understood as subject to significant improvement.

Although TMS has a relatively good spatial accuracy (a few $\mathrm{mm}$ ), there are limitations that impede accurate focal stimulation, such as betweensubject brain variability. The site of stimulation is selected based on the average coordinates of a particular brain region, and the actual location of any region of interest will vary from individual to individual. Accuracy can be improved by using individual subjects' magnetic resonance imaging (MRI) images, as these images can be used in concert with a neuronavigation system to target particular regions on the imaged brain as a best practice. Unfortunately, because MRI scans are often prohibitively costly, most TMS studies (including the example given in this paper) have not had access to neuronavigation.

The differential recruitment of cortical neurons may also contribute to a variability in the effects of cTBS, resulting in potentially opposite effects of both cTBS and iTBS in some individuals. Hamada and colleagues ${ }^{64}$ report finding no overall difference in MEP modulation among 52 participants receiving cTBS versus iTBS to the primary motor cortex. In their study, some participants exhibited a facilitatory response to cTBS and an inhibitory response to iTBS, while others displayed the reverse pattern, and still others showed uniformly inhibitory or excitatory responses to either form of stimulation. Although many other studies have been able to induce the expected response in their overall sample, Hamada and colleagues' results militate for assessing individual differences in responses to cTBS (or iTBS if applicable) in future experimental designs; Hamada et al. provide a detailed description of how MEP latencies may be used to predict a cTBS/iTBS response within subjects ${ }^{64}$.

Achieving the correct coil orientation and positioning for each subject is another effective means of reducing potential variability in the effects of TBS. When available, stereotaxic neuronavigation software that guides the positioning and orientation can help to ensure accurate stimulation. 
In any event, running a large number of subjects in each experimental condition can also help to mitigate potential noise related to differential responsiveness to TBS.

An additional consideration when performing cTBS is how to implement an appropriate control condition. As was done here, cTBS may be contrasted with a sham condition (i.e., pointing the coil away to avoid actual brain stimulation, or lowering the intensity far below threshold). However, as it is conceivable that the cTBS of any region might induce changes in behavior, a common and arguably best practice is to introduce a condition wherein stimulation is delivered to a region thought to be unrelated to the task.

Stimulation of any one region can lead to a spreading activation to regions that are functionally downstream from or proximal to the area of interest, such that causal interpretations of the functional contribution of individual regions must be undertaken with great caution. Relatedly, augmenting cTBS of the PMFC with neuroimaging would importantly clarify the relative effect of the manipulation on the dmPFC versus dACC subcomponents of the pMFC, in addition to the potential collateral enervation of connected regions. At present, it remains unclear whether the observed effects are owed to downregulation of the dACC, dmPFC, or both. In addition to conventional neuroimaging, connectomic analyses might shed light on how pMFC mechanisms articulate with other regions throughout the brain to modulate ideological modes of cognition.

While cTBS of the pMFC to influence high-level social and ideological judgments remains a relatively new and little-tested method, it has been shown to reduce social conformity ${ }^{48}$ and monetary sharing ${ }^{39}$, in addition to the present reductions in group bias and religious belief ${ }^{1}$. While these findings are encouraging, the extent to which cTBS of the PMFC will influence such high-level judgments remains unclear, as is the replicability of previous effects. In the study highlighted here, prospective participants were carefully prescreened to exclude 'hardliners' with respect to both political orientation (strong liberals or conservatives) and religiosity (committed believers or atheists), leaving a sample of relatively moderate individuals. This effort was made to avoid individuals with hardened attitudes, who may produce reflexive responses via pathways distinct from those involved in the process of actively considering ideological positions. While sensible, these screening criteria may have inadvertently introduced a sample bias of some sort related to factors which deter 'middle-of-the-road' individuals from holding firm positions. Where resources allow for larger and more diverse samples, future researchers should include a broad spectrum of political and religious orientations in order to assess potential moderating effects of such individual differences.

In the present design, all participants were reminded of death to establish a context in which participants might be expected to draw on positive religious beliefs as an ideological solution. Although the findings were consistent with this interpretation, as well as with prior work linking religiosity with confidence in the face of physical danger ${ }^{65,66,67,68}$, exposing all participants to a death prime constitutes a serious limitation, as the data cannot reveal whether downregulation of the pMFC would produce comparable effects at baseline, in the absence of a recent threat cue. Further, it is not possible to determine whether the reduction in out-group bias toward the critical immigrant observed following cTBS of the pMFC reflects a muting in the impact of the death prime, a muting in the impact of the ideological conflict posed by the criticism of the US, or an interaction between the two. Thus, future work should incorporate a non-threat control condition to test whether, regardless of the presence of a background threat such as a reminder of death, downregulation of the pMFC reduces group bias or religious belief.

The present threat-induction was chosen because of the link between the prospect of dying and reassuring concepts of the afterlife, and secondarily because mortality-salience induction has been shown to heighten intergroup prejudice ${ }^{69}$. Alternate non-death threat inductions related to challenges in domains pertinent to other social judgments might be comparably employed. Importantly, non-death threat primes have been demonstrated to reliably modulate social judgments ${ }^{14,55,69}$.

Finally, one of the most challenging aspects of this work is that it requires expertise not only in TMS techniques but also in manipulating and measuring relatively abstract constructs such as ideological adherence. Thus, wherever possible, research teams should include investigators with relevant experience in disciplines such as social psychology, political psychology, or anthropology, as well as neuroscience.

\section{Disclosures}

The authors have nothing to disclose.

\section{Acknowledgements}

Colin Holbrook was supported by the U.S. Air Force Office of Scientific Research Award FA9550-115-1-0469.

\section{References}

1. Holbrook, C., Izuma, K., Deblieck, C., Fessler, D.M.T., lacoboni, M. Neuromodulation of group prejudice and religious belief. Social Cognitive and Affective Neuroscience. 11 (3), 387-394 (2016).

2. Allport, G.W. The Nature of Prejudice. Addison-Wesley. Cambridge, MA. (1954).

3. Brewer, M.B. The psychology of prejudice: Ingroup love and outgroup hate? Journal of Social Issues. 55 (3), $429-444$ (1999).

4. Fiske, S.T. What we know now about bias and intergroup conflict, the problem of the century. Current Directions in Psychological Science. 11 (4), 123-128 (2002).

5. Dovidio, J.F., Gaertner, S.L. Intergroup bias. In Handbook of Social Psychology. Edited by Fiske, S.T., Gilbert, D., Lindzey, G., 1084-1121, Wiley. New York, NY (2010).

6. Wrangham, R.W. Evolution of coalitionary killing. American Journal of Physical Anthropology. 110 (29), 1-30 (1999).

7. Neuberg, S.L., Kenrick, D.T., Schaller, M. Evolutionary social psychology. In Handbook of Social Psychology. Edited by Fiske, S.T., Gilbert, D., Lindzey, G., 761-797, Wiley. New York, NY (2010).

8. Hammond, R.A., Axelrod, R. The evolution of ethnocentrism. Journal of Conflict Resolution. 50 (6), $926-936$ (2006).

9. Darwin, C. The descent of man. Appleton. New York, NY(1873).

10. Efferson, C., Lalive, R., Fehr, E. The coevolution of cultural groups and ingroup favoritism. Science. 321 (5897), 1844-1849 (2008). 
11. De Dreu, C.K., Greer, L.L., Van Kleef, G.A., Shalvi, S., Handgraaf, M.J. Oxytocin promotes human ethnocentrism. Proceedings of the National Academy of Sciences of the United States of America. 108 (4), 1262-1266 (2011).

12. Navarrete, C.D., Kurzban, R., Fessler, D.M.T., Kirkpatrick, L. Anxiety and intergroup bias: terror-management or coalitional psychology? Group Processes and Intergroup Relations. 7 (4), 370-397 (2004).

13. McGregor, I., Prentice, M., Nash, K. Anxious uncertainty and reactive approach motivation (RAM) for religious, idealistic, and lifestyle extremes. Journal of Social Issues. 69 (3), 537-563 (2013).

14. Jonas, E. et al. Threat and defense: from anxiety to approach. In Advances in Experimental Social Psychology. Edited by Olson, J.M., Zanna, M.P., 219-286, Academic Press. San Diego, CA (2014).

15. Holbrook, C. Branches of a twisting tree: domain-specific threat psychologies derive from shared mechanisms. Current Opinion in Psychology. 7, 81-86 (2016).

16. Inzlicht, M., McGregor, I., Hirsh, J.B., Nash, K. Neural markers of religious conviction. Psychological Science. 20 (3), $385-392$ (2009).

17. Proulx, T., Inzlicht, M., Harmon-Jones, E. Understanding all inconsistency compensation as a palliative response to violated expectations. Trend in Cognitive Science. 16 (5), 285-291 (2012).

18. Tritt, S.M., Inzlicht, M., Harmon-Jones, E. Toward a biological understanding of mortality salience (and other threat compensation processes). Social Cognition. 6, 715-733 (2012).

19. Klackl, J., Jonas, E., Kronbichler, M. Existential neuroscience: Self-esteem moderates neuronal responses to mortality-related stimuli. Social Cognitive and Affective Neuroscience. 9 (11), 1754-1761 (2014).

20. Luo, S., Shi, Z., Yang, X., Wang, X., Han, S. Reminders of mortality decrease midcingulate activity in response to others' suffering. Social Cognitive and Affective Neuroscience. 9 (4), 477-486 (2014).

21. Etkin, A., Egner, T., Kalisch, R. Emotional processing in anterior cingulate and medial prefrontal cortex. Trend in Cognitive Science. 15 (2), 85-93 (2011).

22. Maier, S. et al. Clarifying the role of the rostral dmPFC/dACC in fear/anxiety: learning, appraisal or expression? PLOS One. 7 (11), e50120 (2012).

23. Rushworth, M.F., Buckley, M.J., Behrens, T.J., Walton, M.E., Bannerman, D.M. Functional organization of the medial frontal cortex. Current Opinion in Neurobiology. 17 (2), 220-227 (2007).

24. Shima, K., Tanji, J. Role for cingulate motor area cells involuntary movement selection based on reward. Science. 282 (5392), 1335-1338 (1998).

25. Bush, G. et al. Dorsal anterior cingulate cortex: A role in reward-based decision making. Proceedings of the National Academy of Sciences of the United States of America. 99 (1), 523-528 (2002).

26. Ridderinkhof, K.R., Ullsperger, M., Crone, E.A., Nieuwenhuis, S. The role of the medial frontal cortex in cognitive control. Science. 306 (5695), 443-447 (2004).

27. Han, S., Qin, J., Ma, Y. Neurocognitive processes of linguistic cues related to death. Neuropsychologia. 48 (12), $3436-3442$ (2010).

28. Shi, Z., Han, S. Transient and sustained neural responses to death-related linguistic cues. Social Cognitive and Affective Neuroscience. 8 (5), 573-578 (2013).

29. Eisenberger, N.I. Broken hearts and broken bones: a neural perspective on the similarities between social and physical pain. Current Directions in Psychological Science. 21 (1), $42-47$ (2012).

30. Spengler, S., von Cramon, D.Y., Brass, M. Resisting motor mimicry: Control of imitation involves processes central to social cognition in patients with frontal and temporo-parietal lesions. Social Neuroscience. 5 (4), 401-416 (2010).

31. Taylor, J.J., Borckardt, J.J., George, M.S. Endogenous opioids mediate left dorsolateral prefrontal cortex rtms-induced analgesia. Pain. 153 (6), 1219-1225 (2012).

32. Zaki, J., Ochsner, K.N., Ochsner, K. The neuroscience of empathy: Progress, pitfalls and promise. Nature Neuroscience. 15 (5), 675-680 (2012).

33. Hein, G., Lamm, C., Brodbeck, C., Singer, T. Skin conductance response to the pain of others predicts later costly helping. PLOS One. 6 (8), e22759 (2011).

34. Hein, G., Silani, G., Preuschoff, K., Batson, C.D., Singer, T. Neural responses to ingroup and outgroup members' suffering predict individual differences in costly helping. Neuron. 68 (1), 149-160 (2010).

35. Ma, Y., Wang, C., Han, S. Neural responses to perceived pain in others predict real life monetary donations in different socioeconomic contexts. Neurolmage. 57 (3), 1273-1280 (2011).

36. Reynolds Losin, E.A., lacoboni, M., Martin, A. Cross, K., Dapretto, M. Race modulates neural activity during imitation. Neurolmage. 59 (4), 3594-3603 (2012).

37. Cross, K.A., Torrisi, S., Reynolds Losin, E.A., lacoboni, M. Controlling automatic imitative tendencies: Interactions between mirror neuron and cognitive control systems. Neurolmage. 83, 493-504 (2013).

38. Amodio, D.M., Frith, C.D. Meeting of minds: the medial frontal cortex and social cognition. Nature Reviews Neuroscience. 7, 268-277 (2006).

39. Christov-Moore, L., Sugiyama, T., Grigaityte, K., lacoboni, M. Increasing generosity by disrupting prefrontal cortex. Social Neuroscience. 12 (2), 174-181 (2017).

40. Downar, J., Blumberger, D.M., Daskalakis, Z.J. The neural crossroads of psychiatric illness: an emerging target for brain stimulation. Trends in Cognitive Sciences. 20 (2), 107-120 (2016).

41. Cho, S.S. et al. Investing in the future: stimulation of the medial prefrontal cortex reduces discounting of delayed rewards. Neuropsychopharmacology. 40, 546-553 (2015).

42. Izuma, K. The neural basis of social influence and attitude change. Current Opinion in Neurobiology. 23 (3), $456-462$ (2013).

43. van Veen, V., Krug, M.K., Schooler, J.W., Carter, C.S. Neural activity predicts attitude change in cognitive dissonance. Nature Neuroscience. 12 (11), 1469-1474 (2009).

44. Izuma, K. et al. Neural correlates of cognitive dissonance and choice-induced preference change. Proceedings of the National Academy of Sciences of the United States of America. 107 (51), 22014-22019 (2010).

45. Izuma, K. et al. A causal role for posterior medial frontal cortex in choice-induced preference change. Journal of Neuroscience. 35 (8), 3598-3606 (2015).

46. Klucharev, V., Hytonen, K., Rijpkema, M., Smidts, A., Fernandez, G. Reinforcement learning signal predicts social conformity. Neuron. 61 (1), 140-151 (2009).

47. Izuma, K., Adolphs, R. Social manipulation of preference in the human brain. Neuron. 78 (3), 563-573 (2013). 
48. Klucharev, V., Munneke, M.A., Smidts, A., Fernández, G. Downregulation of the posterior medial frontal cortex prevents social conformity. Journal of Neuroscience. 31 (33), 11934-11940 (2011).

49. Ullsperger, M., Volz, K.G., Cramon, D.Y. A common neural system signaling the need for behavioral changes. Trends in Cognitive Sciences. 8, 445-446 (2004).

50. Fregni, F., Pascual-Leone, A. Technology insight: noninvasive brain stimulation in neurology-perspectives on the therapeutic potential of rTMS and tDCS. Nature Reviews Neurology. 3 (7), 383 (2007).

51. Rossi, S., Hallett, M., Rossini, P.M., Pascual-Leone, A. Safety, ethical considerations, and application guidelines for the use of transcranial magnetic stimulation in clinical practice and research. Clinical Neurophysiology. 120 (12), 2008-2039 (2009).

52. Hallett, M. Transcranial magnetic stimulation and the human brain. Nature. 406 (6792), 147 (2000).

53. Huang, Y.Z., Edwards, M.J., Rounis, E., Bhatia, K.P., Rothwell, J.C. Theta burst stimulation of the human motor cortex. Neuron. 45 (2), 201-206 (2005).

54. McGregor, H. et al. Terror management and aggression: evidence that mortality salience motivates aggression against worldview threatening others. Journal of Personality and Social Psychology. 74 (3), 590-605 (1998).

55. Holbrook, C., Sousa, P., Hahn-Holbrook, J. Unconscious vigilance: worldview defense without adaptations for terror, coalition or uncertainty management. Journal of Personality and Social Psychology. 101 (3), 451-466 (2011).

56. Bakker, N. et al. rTMS of the dorsomedial prefrontal cortex for major depression: safety, tolerability, effectiveness, and outcome predictors for $10 \mathrm{~Hz}$ versus intermittent theta-burst stimulation. Brain Stimulation. 8, 208-215 (2015).

57. Dunlop, K. et al. MRI-guided dmPFC-rTMS as a treatment for treatment-resistant major depressive disorder. Journal of Visualized Experiments. (102), e53129 (2015).

58. Klem, G.H., LuĖders, H.O., Jasper, H.H., Elger, C. The ten-twenty electrode system of the International Federation. Electroencephalography and Clinical Neurophysiology. 52 (3), 3-6 (1999).

59. Bush, G, Shin, L.M. The Multi-Source Interference Task: an fMRI task that reliably activates the cingulo-frontal-parietal cognitive/attention network. Nature Protocols. 1 (1), 308-313 (2006).

60. Rosenblatt, A., Greenberg, J., Solomon, S., Pyszcynski, T., Lyon, D. Evidence for terror management theory: I. The effects of mortality salience on reactions to those who violate or uphold cultural values. Journal of Personality and Social Psychology. 57 (4), 681-690 (1989).

61. Watson, D., Clark, L.A. The PANAS-X: Manual for the Positive and Negative Affect Schedule-Expanded Form. The University of lowa (1994).

62. Greenberg, J., Pyszczynski, T., Solomon, S., Simon, L., Breus, M. Role of consciousness and accessibility of death-related thoughts in mortality salience effects. Journal of Personality and Social Psychology. 67 (4), 627-637 (1994).

63. Jong, J., Halberstadt, J., Bluemke, M. Foxhole atheism, revisited: The effects of mortality salience on explicit and implicit religious belief. Journal of Experimental Social Psychology. 48 (5), 983-989 (2012).

64. Hamada, M., Murase, N., Hasan, A., Balaratnam, M., Rothwell, J.C. The role of interneuron networks in driving human motor cortical plasticity. Cerebral Cortex. 23 (7), 1593-1605 (2012).

65. Pollack, J., Holbrook, C., Fessler, D.M.T., Sparks, A.M., Zerbe, J.G. May God guide our guns: Visualized supernatural aid heightens team confidence in a paintball battle simulation. Human Nature. In Press (2018).

66. Holbrook, C., Pollack, J., Zerbe, J.G., Hahn-Holbrook, J. Perceived supernatural support enhances battle confidence: A knife combat field study. Religion, Brain \& Behavior. (2018).

67. Holbrook, C., Fessler, D.M.T., Pollack, J. With God on your side: Religious primes reduce the envisioned physical formidability of a menacing adversary. Cognition. 146, 387-392 (2016).

68. Kupor, D.M., Laurin, K., Levav, J. Anticipating divine protection? Reminders of god can increase nonmoral risk taking. Psychological Science. 26, .374-384 (2015).

69. Holbrook, C. Branches of a twisting tree: Domain-specific threat psychologies derive from shared mechanisms. Current Opinion in Psychology. 7, 81-86 (2016). 